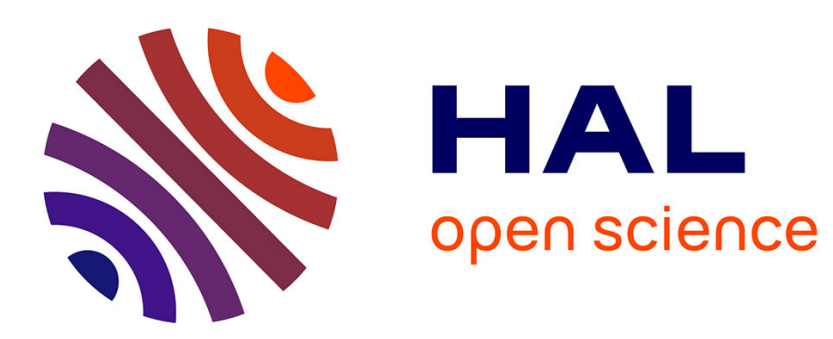

\title{
Les Concours de beauté à Tahiti. La fabrication médiatisée d'appartenances territoriale, ethnique et de genre \\ Laura Schuft
}

\section{- To cite this version:}

Laura Schuft. Les Concours de beauté à Tahiti. La fabrication médiatisée d'appartenances territoriale, ethnique et de genre. CORPS : Revue Interdisciplinaire, 2012, $\mathrm{N}^{\circ} 10$ (1), pp.133-142. 10.3917/corp1.010.0133 . halshs-02944113

\section{HAL Id: halshs-02944113 \\ https://shs.hal.science/halshs-02944113}

Submitted on 21 Sep 2020

HAL is a multi-disciplinary open access archive for the deposit and dissemination of scientific research documents, whether they are published or not. The documents may come from teaching and research institutions in France or abroad, or from public or private research centers.
L'archive ouverte pluridisciplinaire HAL, est destinée au dépôt et à la diffusion de documents scientifiques de niveau recherche, publiés ou non, émanant des établissements d'enseignement et de recherche français ou étrangers, des laboratoires publics ou privés. 
Cet article, ici en version auteur, a paru dans un numéro spécial, coordonné par Chantal CRENN et Simona TERSIGNI, de la Revue Corps. La référence complète est :

SCHUFT, Laura (2012). Les Concours de beauté à Tahiti. La fabrication médiatisée d'appartenances territoriale, ethnique et de genre, Corps, $\mathrm{n}^{\circ} 10$ (Corps en relations interethniques : migrations, identifications et hiérarchisations), pp.133-142.

\section{Les Concours de beauté à Tahiti. La fabrication médiatisée d'appartenances territoriale, ethnique et de genre}

\section{Laura Schuft}

Abondants et abondamment médiatisés, les concours de beauté en Polynésie française, notamment à Tahiti, s'organisent au sein de maintes institutions de la société - des établissements scolaires aux hôtels et journaux, des villages aux îles. Cet article se propose en réflexion sur la manière dont la médiatisation des concours de beauté à Tahiti, le centre administratif et l'île la plus peuplée de cette Collectivité d'Outre Mer, participe à tisser et à renforcer les associations entre son physionomie corporelle et son appartenance territoriale, ethnique et de genre. Si certains travaux ont soulevé des modalités de représenter une nation à travers des mises en scène stéréotypées - racisées et genrées (gendered) - des concours de beauté (Banet-Wieser, 1999 ; Cunin, 2004), cet article se focalise plus particulièrement sur les processus d'attribution aux corps d'appartenances sociales imbriquées - ethnique, genrée et territoriale - à travers leur médiatisation locale.

Après une présentation du cadre théorique et méthodologique de ce travail suivi par une présentation du contexte sociohistorique tahitien, cet article considère en premier lieu la représentation spécifique de corps (féminin, « exotique », jeune) qui est réappropriée comme métaphore de Tahiti dans certains concours de beauté. Cette réappropriation du mythe de la vahine a lieu malgré l'influence coloniale (masculine, occidentale) sur la genèse de cette représentation. Si l'image de Tahiti comme vahine constitue un enjeu touristique et politique pour certains acteurs de la Polynésie française, d'autres concours de beauté, également médiatisés et populaires au niveau local, mettent en scène d'autres articulations de genre, de physionomie et de culture. Ces corps en scène sont également spécifiés (en termes de « race » ou de genre) mais ne sont pas, quant à eux, érigés en représentations métaphoriques de Tahiti. Cet article considère donc en deuxième lieu les processus de compartimentage et de racialisation des appartenances sociales qui sont véhiculés par la médiatisation de l'ensemble des concours de beauté.

\section{CADRE THEORIQUE ET METHODOLOGIQUE}

La réflexion présentée ici se nourrit de travaux sociologiques américains et français dans un champ théorique sur «l'intersectionnalité » qui traite de la manière dont l'ethnicité, le genre et le statut socioéconomique s'articulent, et dont leurs représentations et oppressions sociales s'imbriquent (cf. Dorlin, 2009). Ce cadre théorique est conjugué aux travaux depuis Goffman (1973) qui analysent les manières dont les mises en scène des appartenances sociales participent à (re)produire ces dernières (cf. Zaidman, 2009). Il s'agit de considérer ici les modes de (re)production et de compartimentage des appartenances sociales imbriquées ${ }^{1}$ à travers ces mises en scène. Car, selon les théories interactionnistes depuis Goffman, les concours de beauté sont susceptibles de jouer un rôle majeur dans la propagation de 
représentations sociales. D'une part, les critères de distinction entre catégories sociales sont plus que socialement normés; ils sont formalisés et fortement codés. D'autre part, leur médiatisation amplifie le nombre d'observateurs à des échelles sociales bien plus larges que celles des mises en scène de l'interaction sociale quotidienne. La médiatisation des concours de beauté peut ainsi amplifier la (re)production des représentations sociales imbriquées.

Par ailleurs, depuis le travail de MacCannell (1973), des travaux anthropologiques et sociologiques explorent la manière dont la médiatisation et le marketing touristiques de «l'authenticité » culturelle, visant à attirer les touristes qui seraient en «quête d'authenticité », incitent la mise en scène des populations elles-mêmes dans la promotion de leur territoire en tant que destination touristique (cf. Rinaudo \& Cunin, 2008). Les concours «Miss Tahiti» ou «Miss Polynésie française», censés fabriquer «l'ambassadrice de la Polynésie française », sont subséquemment considérés dans leur contexte de globalisation et de compétition entre destinations touristiques. Il s'agit de considérer la manière dont certaines des mises en scène - de corps spécifiés - visent à donner à voir une image spécifique de la région qui est racisée et genrée. Bien que de telles images de la région soient promues à des fins notamment économiques et touristiques, elles semblent participer à (re)définir et à réifier des appartenances ethniques genrées.

L'analyse se base sur une revue de la presse récente (2006-2009) en Polynésie française. Il s'agit, d'une part, des brochures, articles et vidéos touristiques tahitiens en ligne, en particulier ceux créés par Tahiti Tourism, un consortium financé par le gouvernement local ${ }^{2}$, mais aussi ceux créés par d'autres sites visant la promotion internationale de l'image de Tahiti. L'analyse se base, d'autre part, sur des articles de presse locaux ayant pris comme objet les concours de beauté ou l'appel à y candidater; les sites officiels des concours ; et des vidéos des concours eux-mêmes. Ces approches théoriques et méthodologiques se rajoutent aux observations durant cinq années vécues à Tahiti, lieu de mes recherches doctorales sur un thème annexe (Schuft, 2010).

\section{LA VAHINE : LONGTEMPS REPRESENTATIVE DE TAHITI DANS LE REGARD OCCIDENTAL}

Des représentations spécifiques de «femmes polynésiennes », sous la désignation de vahine, avaient déjà atteint l'Europe à la fin du $18^{\mathrm{e}}$ siècle, à l'instar des écrits par les premiers navigateurs européens dans les îles polynésiennes. (Vahine veut dire «femme» en langue tahitienne, mais est généralement employé pour signifier « femme polynésienne », notamment en lien avec le mythe de la vahine). Depuis, les regards et les interprétations masculins et euro-centriques sur les femmes de Tahiti en ont fait l'objet de désir masculin et européen, comme en témoigne une longue liste de littérature et de films qui reprennent cette représentation singulière des femmes polynésiennes exotisées. Cette représentation singulière de la vahine, vénérée par les marins ou décriée par les missionnaires, a si souvent été reprise dans les écrits sur Tahiti qu'elle est devenue en quelque sorte une métaphore du lieu.

La construction littéraire européenne - et masculine - de Tahiti assimile cette île à une femme (Margueron, 1989) - plus spécifiquement, à une femme ethnicisée et érotisée. Selon Todorov, cette formule est l'invention de Loti, qui «fait coïncider exotisme et érotisme : la femme est exotique, l'étranger est érotique [...] (le visiteur aime le pays étranger comme l'homme aime la femme, et inversement) (Todorov, 1989: 416-7). Avec Le Mariage de Loti à Tahiti, publié en 1880 quand ces îles deviennent officiellement une colonie française, Loti lance l'habitude de marier dans la littérature coloniale l'image de femme - ethnicisée, exotisée et érotisée - avec un désir masculin, hétérosexuel et occidental.

L'image qui superpose «femme» et «Tahiti» a également pénétré certaines représentations politiques des territoires, comme le montre l'ouvrage historique Le Mariage franco-tahitien (Toullelan \& Gille, 1992). Soulignant les bienfaits du protecteur colonial 
métropolitain dans son histoire «matrimoniale » avec la Polynésie française, cet ouvrage se sert de l'ordre supposé «naturel » de domination et protection masculine dans le couple pour justifier d'une domination ethno-politique «naturelle» et bienveillante d'une région (masculinisée) sur l'autre (féminisée). Le mythe de la vahine et la représentation féminisée de la région ont ainsi servi l'idéologie coloniale en transposant les rapports de domination dans le genre sur les rapports interrégionaux.

\section{REAPPROPRIATION MEDIATIQUE DU MYTHE DE LA VAHINE}

A croire les cartes postales ornées des images de la vahine ou les interminables concours de Miss Tahiti, Tahiti est encore représentée comme une «femme exotique», notamment selon l'instrumentalisation du mythe de la vahine par des stratégies économiques ou politiques. Plusieurs produits exportés et consommés localement - les logos de la bière ou du monoï - n'hésitent pas, par exemple, à s'emparer de cette image des corps des femmes dans un «marketing de la différence » (Rinaudo \& Cunin, 2008), qui vise à marquer la spécificité d'un lieu dans un contexte de globalisation et de compétitivité économique et touristique interrégionale. Les représentations des corps féminins et «polynésiens » semblent, selon les images et discours touristiques et politiques médiatisés, un enjeu du marketing touristique, le tourisme étant la première source de PIB de la Collectivité après les transferts, bien importants, de l'Etat français ${ }^{3}$.

Les concours de Miss Tahiti s'inscrivent dans ce double processus, d'une réappropriation locale du mythe de la vahine et de son établissement comme représentatif de la culture et du territoire de Tahiti. Par «mythe de la vahine », il est entendu la représentation singulière, imaginaire et renommée des femmes «polynésiennes » depuis les premiers écrits de la part des hommes européens à leur sujet. La mise en scène de la soirée d'élection Miss Tahiti 2008 est exemplaire de la réappropriation du mythe de la vahine par certains concours de beauté. Le thème de cette soirée était justement la recréation de l'épopée de la Bounty de 1789, dont les vahine figurent en premier plan des cinq reproductions en film. Dans cette mise en scène, "les invités auront l'impression d'être embarqués à bord de la Bounty et [...] de débarquer sur une île. Une île peuplée de "vahine", toutes candidates au titre le plus prestigieux qui soit sous ces latitudes ${ }^{4}$. L'image de la «vahine» comme objet de désir masculin et occidental est ainsi reproduite, soulignant ce regard sur l'île et sur les femmes, ainsi que la mise à l'écart littéraire habituelle des hommes dont l'île était également peuplée (cf. Cerf, 2005).

Miss Tahiti est non seulement appelée à incarner une féminité spécifique reliée au regard masculin et européen depuis la fin du $18^{\mathrm{e}}$ siècle, mais la gagnante est également désignée dans les médias comme «ambassadrice de la Polynésie française » ou «de son pays $»^{5}$. Sa représentativité est rappelée par les candidates et le site officiel du concours, qui affirment que Miss Tahiti est «l'ambassadrice d'un peuple et de sa culture » ou « l'incarnation même de la beauté polynésienne ». Pour la Présidente du Comité de Miss Tahiti, «la beauté de la femme polynésienne » représente Tahiti en raison de leurs caractéristiques partagées : «la joie de vivre de Tahiti ${ }^{6}$. Les candidates au concours en 2009 font écho au rôle de la gagnante de «représenter» ou «incarner» la beauté et la culture de son «pays» ou «peuple» (www.misstahiti.com). Un nouveau concours créé en 2009, Miss Polynésie française, accentue la notion de représentativité territoriale, ainsi que ses enjeux touristiques. Cette accentuation est accomplie non seulement par son titre, Miss Polynésie française, plus inclusif de l'ensemble des îles de la Collectivité, mais également par la nomination d' « ambassadrices »- « du Tourisme » et « de la Culture »- à la place de dauphines.

A cette mise en scène du mythe de la «féminité polynésienne »- en tant que représentation de Tahiti - on peut remarquer la volonté de mettre en scène une «naturalité » 
des caractéristiques qui y sont attribuées. Les candidates du concours Miss Tahiti en 2009, selon le site officiel du concours, expriment la nécessité que la gagnante soit «belle et dotée d'un charme exotique et naturel », qu'elle soit «synonyme de beauté et d'exotisme ». Sur l'île de Tahiti - à travers les corps féminisés et ethnicisés - se projettent donc des attributs d'exotisme et de beauté «naturels », «innés» selon une autre candidate. On rejoint ici l'hypothèse d'Anibal Quijano, qui avance que le fait de percevoir les notions de 'corps' et de 'race' comme 'naturels', en opposition avec 'esprit' et 'modernité', est au cœur de la perspective euro-centrique qui conditionne les relations mondiales d'aujourd'hui (Quijano, 2007 : 117). Autant la notion de 'race' que celle de 'sexe' sont «considérées comme des catégories 'naturelles' », notion qui est inséparable de l'histoire des rapports sociaux de pouvoir - coloniaux et sexistes - au cours de laquelle ces catégories sociales se sont construites (Guillaumin, 1992 : 10). Le fait de féminiser et d'ethniciser le territoire s'inscrit ainsi dans ce processus colonial de naturalisation de l'Autre.

APPARTENANCES COMPARTIMENTEES, RACIALISEES ET ANCREES DANS LE CORPS

La popularité des concours de beauté à Tahiti ne se limite point aux grands concours les plus médiatisés et subventionnés. De multiples concours populaires et fréquents font également appel à des corps spécifiés pour mettre en scène des appartenances imbriquées à Tahiti, selon les appartenances ethniques, de genre et de sexualité. De multiples concours sont nés depuis le concours Miss Tahiti (depuis 1960) : Miss Dragon (concours féminin chinois, depuis 1970), Mr. Tahiti (concours masculin polynésien, depuis 2001), Miss Vahine Tane (concours travesti, depuis 1997) et plus récemment Miss Popa'a (concours féminin européen, depuis 2004) ${ }^{7}$. Chacun interroge les manières dont les appartenances ethniques, de genre et de sexualité s'enracinent socialement dans le corps. Le lien normatif entre le corps et l'appartenance ethnique genrée est particulièrement interrogé par le concours Miss Vahine Tane qui, tout en reproduisant les mêmes appartenances imbriquées particulières (ethnique, territoriale, culturelle, de genre) en vigueur dans les concours de Miss Tahiti, fait appel spécifiquement aux corps de sexe masculin dans la mise en scène du genre féminin. Considérons ici ces processus de différenciation corporelle et de compartimentages identitaires que soulignent les différents concours de beauté.

Tout d'abord, l'accent sur un lien supposé entre le corps et une appartenance « raciale » ou biologique apparaît comme primordial dans tous les concours. Sur le site officiel du concours Miss Polynésie française, les «critères de sélection » pour «devenir Miss » sont explicites et débutent avec l'obligation d'être «de souche Polynésienne (au moins 1 des 2 parents) $\gg$. Pour le concours d'une commune de Tahiti, Miss Mahina, on exige également d' «être née de souche polynésienne » ${ }^{9}$. L'usage du terme «souche », débattu en France métropolitaine pour le sens racialisant qui lui est attribué, ancre les critères de sélection dans la mise en lien entre le corps et une appartenance "raciale », et avance que certains résidents de la Polynésie française serait plus «vrais » ou «polynésiens » que d'autres. On remarque par exemple qu'à la place du lieu de naissance ou du temps vécu dans la région, "souche » sert de rappeler les exigences physionomiques du concours et la croyance dans une «race» biologique qui permettrait de distinguer entre appartenances sociales.

L'application des critères physionomiques dans la sélection des candidats révèle également l'ambigüité des catégorisations sociales, notamment lorsqu'il s'agit d'incorporer le métissage pour lequel Tahiti est si connu (cf. par exemple Toullelan \& Gilles, 1992). Notamment, si le métissage physionomique et culturel apparaît comme un critère primordial dans les concours Miss Tahiti, ce métissage peut exclure. Le métissage physionomique promu par ce concours est à fort tendance «polynésien »-« européen », les dauphines étant plutôt de 
couleur «blanche » et de traits occidentaux. Certaines participantes aux concours Miss Popa'a ou Miss Dragon expliquent leur candidature par l'impossibilité de participer à Miss Tahiti n'étant pas métissée «polynésienne » ou encore étant «trop typées chinoises » ${ }^{10}$. Dans un «Espace de discussion citoyen » du site de l'Assemblée de la Polynésie française, hébergeant une centaine de réactions au sujet de «Miss Popa'a - Miss Ségrégation ? »depuis avril 2005 (un an après la première élection de Miss Popa'a), de nombreux intervenants soulignent l'impossibilité pour une jeune femme ayant vécu toute sa vie à Tahiti de participer à l'élection de Miss Tahiti en raison de sa «couleur de peau». Selon leurs propos, les concours Miss Popa'a et Miss Dragon apparaissent comme des réponses à l'exclusion engendrée par les concours Miss Tahiti qui ancrent dans des corps spécifiques des standards de beauté et d'appartenance à la fois territoriale, ethnique et de genre.

L'ensemble des concours sont à ce titre des affirmations d'appartenances imbriquées et compartimentées. Si les corps mis en avant par les différents concours ne sont pas tous tenus comme «représentatifs » de Tahiti (tel qu'inventé par la logique coloniale et repris par les mises en scène touristiques), chaque concours revendique et valorise une combinaison spécifique d'appartenances sociales multiples, à travers des critères spécifiques de beauté corporelle. La formalisation de ces critères physionomiques révèle - tout en propageant - des modalités normatives de dessiner des «frontières » (Barth, 1969) entre catégories sociales qui se veulent « naturelles » ou « raciales ».

\section{CONCLUSION}

Les concours de beauté à Tahiti révèlent, à travers les discours médiatisés et les images à leur sujet, de façons sociales - non exhaustives - de composer des critères corporels et performatifs des appartenances sociales imbriquées à Tahiti. Si les concours participent à ce titre à y (re)produire les appartenances imbriquées et compartimentées, ils en révèlent également les tensions. Car les «frontières »sont floues et les définitions, évolutives, font l'objet de négociations et de débats.

Une seule combinaison d'appartenances fournit une «ambassadrice » de la Collectivité, dont la gagnante, soigneusement sélectionnée, représente par son propre corps le territoire et sa culture. Féminisée et ethnicisée, l'image de Tahiti est alimentée par la réappropriation et la recomposition du regard masculin et occidental depuis la fin du $18^{\mathrm{e}}$ siècle sur ce territoire et ses femmes. Comme dans des concours de beauté féminine en Colombie, «la mise en scène du corps de la femme est porteuse du récit de la [région] sur elle-même » (Cunin, 2004 : 167), qu'elle souhaite donner à voir autant à elle-même qu'à ceux adressés dans ses discours touristiques. Le récit tahitien, dans sa recomposition d'aujourd'hui véhiculée par la médiatisation des concours de beauté, unit folklore et modernité à travers la mise en scène des corps des femmes. Quant aux maints autres concours de beauté, qui affirment diverses appartenances imbriquées en s'appuyant sur des caractéristiques corporelles, ils mettent en évidence les modalités de compartimentage des appartenances locales, et l'appui sur des symboles corporels.

Les réflexions et les analyses abordées ici ouvrent de nombreuses questions sociologiques notamment concernant les effets sociaux de ces concours, dont le rayonnement local est important en raison de leur médiatisation. Il reste à approfondir dans quelle mesure et de quelle manière les représentations identitaires véhiculées par ces mises en scène médiatisées sont appropriées, recomposées ou résistées au cours des mises en scène de la vie quotidienne à Tahiti, à travers les discours et les interactions sociales des candidats euxmêmes ainsi que des jeunes habitants de Tahiti.

Enfin, une question qui demeure sans réponse est celle de savoir quelle signification attribuer à l'absence de certaines catégories imbriquées dans ces mises en scène de la beauté 
corporelle à Tahiti. Dans cette Collectivité fortement marquée par les rapports de pouvoir (post)coloniaux, il serait intéressant de considérer de quelle manière les «absences »notamment des hommes «popa'a » et des hommes «chinois »-s'inscrivent dans les rapports de pouvoir entre catégories sociale, et dans l'attribution ou la négation - aux catégories imbriquées de classe, d'ethnicité, de genre ou de sexualité - de désirabilité sociale et sexuelle.

\section{BIBLIOGRAPHIE}

Banet-Wieser S. 1999, The Most Beautiful Girl in the World: Beauty Pageants and National Identity, Berkeley, University of California Press.

Barth F. 1969, Les groupes ethniques et leurs frontières, traduit dans Poutignat P. \& Streiff-Fenart J. 1996, Théories de l'ethnicité, Paris, PUF.

Cerf P. 2005, «La domination des femmes à Tahiti : des violences envers les femmes au discours du matriarcat », Thèse de doctorat, Université de la Polynésie Française.

Cunin E. 2004, Métissage et multiculturalisme en Colombie (Carthagène). Le "noir » entre apparences et appartenances, Paris, l'Harmattan.

Dorlin E. (dir.) 2009, Sexe, Race, Classe, pour une épistémologie de la domination, Paris, PUF.

Goffman E. 1973, La mise en scène de la vie quotidienne, Paris, Editions de Minuit.

Guillaumin C. 1992, Sexe, race et pratique du pouvoir, Paris, Côté-femmes.

Kergoat D. 2009, « Dynamique et consubstantialité des rapports sociaux », dans Dorlin E. (dir.), Sexe, Race, Classe, pour une épistémologie de la domination, Paris, PUF, pp.111-126.

MacCannell D. 1973, « Staged Authenticity : Arrangements of Social Space in Tourist Settings » dans American Journal of Sociology, $\mathrm{n}^{\circ} 3$, vol. $79: 589-603$.

Margueron D. 1989, Tahiti dans toute sa littérature, Paris, l'Harmattan.

Quijano A. 2007, «"Race" et colonialité du pouvoir » dans Mouvements (« Qui a peur du postcolonial ? Dénis et controverses), $\mathrm{n}^{\circ} 51: 111-118$.

Rinaudo C. \& Cunin E. 2008, «Consommer la ville en passant : visites guidées et marketing de la différence à Cartagena de Indias (Colombie) » dans Espaces et Sociétés, n 135 : 139-156.

Schuft L. 2010, « Couples 'métropolitain' - 'polynésien' à Tahiti. Enjeux de l'ethnicité, du genre et du statut socioéconomique dans un contexte postcolonial », Thèse de doctorat, Université de Nice-Sophia Antipolis.

Todorov T. 1989, Nous et les autres, Paris, Seuil.

Toullelan P.-Y. \& Gille B. 1992, Le Mariage Franco-Tahitien, Papeete, Polymages-Scoop.

\footnotetext{
${ }^{1}$ «Appartenances imbriquées » fait référence à la multiplicité de caractéristiques sociales prises en compte dans la catégorisation sociale de personnes : dont notamment le genre et le «groupe ethnique ». Par exemple, le fait d'être catégorisée comme «femme polynésienne » est profondément différent (en termes des représentations et des discriminations sociales) que d'être catégorisé comme «homme polynésien» ou comme «femme métropolitaine ».

${ }^{2}$ Site du Ministère du Tourisme, 'CM du lundi 28 décembre / Subvention promotion touristique / Gie Tahiti Tourisme (28-12-2009)', URL: http://www.tourisme.gov.pf/7555-CM-du-lundi-28-decembre-Subventionpromotion-touristique-Gie-Tahiti-Tourisme.html. Consulté le 22-02-2011.

${ }^{3}$ Cf. le Rapport économique sur la Polynésie française, juin 1999, publié par Bank of Hawaii. Les dépenses publiques en Polynésie française, personnels et transferts inclus, sont évaluées à 55\% du PIB («La gestion des
} 


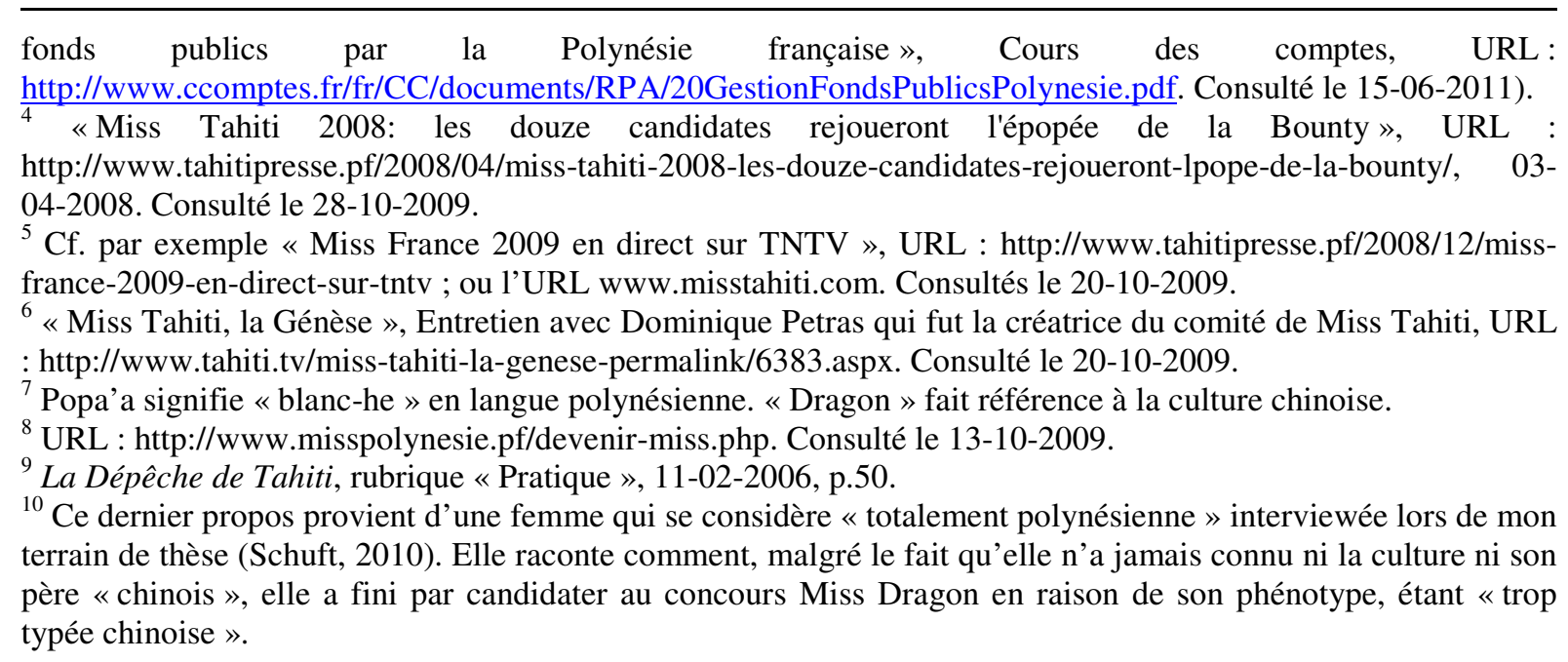

